\title{
Nuevos registros de digeneos en Podocnemis spp. (Testudines, Podocnemididae) de lquitos, Perú
}

\section{New records of digeneans from Podocnemis spp. (Testudines, Podocnemididae) from lquitos, Peru}

\author{
Manuel Tantaleán ${ }^{1}$, Nofre Sánchez² y Oscar Pineda Catalan ${ }^{3}$
}

\begin{abstract}
1 Escuela de Post Grado. Facultad de Ciencias Biológicas. Universidad Nacional Mayor de San Marcos. Lima, Perú.

E-mail: mtantaleanv@hotmail.com 2 IVITA - Iquitos. Facultad de Medicina Veterinaria Universidad ricional Mayc

E-mail: nofresp@hotmail.com

3 Columbia University, Ecology, Evolution and Environmental Biology Department, 1200 Amsterdam Ave. New York, NY, USA. American Ave Museum of Natural History, Sackler Institute for Comparative Genomics, Central Park West \& 79 Street, New York, NY, USA. E-mail: op2101@columbia.edu

Presentado: $\quad 30 / 06 / 2010$ Aceptado: $\quad 23 / 11 / 2010$ Publicado online: $23 / 06 / 2011$
\end{abstract}

\section{Resumen}

En el mercado de Belén (Iquitos, Perú) se obtuvieron ocho tractos digestivos de Podocnemis expansa y 18 de $P$. unifilis los que fueron analizados en búsqueda de parásitos, detectándose la presencia de digeneos y nematodos. Solo se estudió los digeneos, los que fueron identificados como Nematophila grandis (Diesing, 1839) Travassos, 1934, Halltrema avitellina Lent \& Freitas, 1939, Podocnemitrema papillosum Alho \& Vicente, 1964 y Telorchis hagmanni Lent \& Freitas, 1937. Halltrema avitellina, Podocnemitrema papillosum y Telorchis hagmanni son nuevos registros para el Perú.

Palabras clave: Digenea, Podocnemis expansa, Podocnemis unifilis, Perú.

\section{Abstract}

We obtained 8 digestive tract of the turtle Podocnemis expansa and 18 of $P$. unifilis from the Belen market (Iquitos, Peru). Only digeneans were studied and identified. Four species were found: Nematophila grandis (Diesing, 1839) Travassos, 1934, Halltrema avitellina Lent \& Freitas, 1939, Podocnemitrema papillosum Alho \& Vicente, 1964 and Telorchis hagmanni Lent \& Freitas, 1937. Halltrema avitellina, Podocnemitrema papillosum and Telorchis hagmanni are new records from Peru.

Keywords: Digeneans, Podocnemis expansa, Podocnemis unifilis, Peru.

\section{Introduccion}

A pesar de su importancia económica y biológica, en el Perú no es muy conocida la diversidad de digeneos en Podocnemis spp., las publicaciones parasitológicas sobre ellas mayormente se refieren a nemátodes (Tantaleán et al. 1983, Tantaleán 1998, Sarmiento et al. 1999, Sánchez et al. 2006, Salízar \& Sánchez 2007). En la actualidad, la mayoría de estudios parasitológicos en animales de vida silvestre se llevan a cabo utilizando medios no invasivos, lo que dificulta la identificación de los parásitos cuyos huevos son eliminados con las heces. Sin embargo, en el mercado de Iquitos se comercializa la carne de tortugas, entre otros animales, por lo que se pueden adquirir los órganos internos.

\section{Material y métodos}

Para el presente trabajo, se estudiaron tractos digestivos de 26 tortugas colectadas entre los meses de julio y agosto de 2008, procedentes de Iquitos, Perú; 8 fueron de la especie Podocnemis expansa (Schweigger, 1812) y 18 de Podocnemis unifilis Troschel, 1848.

Los digeneos obtenidos se lavaron en suero fisiológico, se prensaron entre 2 láminas portaobjetos y procesaron para tinción con carmín acético de Semichon de acuerdo a la técnica convencional. Antes del montaje, a cada espécimen coloreado y clarificado se le retiró parte del tegumento y músculos de las caras ventral y dorsal con la finalidad de visualizar mejor los órganos que forman el complejo genital. Las medidas se dan en milímetros salvo que se indique otra cosa, anotando primero el promedio y luego el rango entre paréntesis.

Algunos de los especímenes se depositaron en la Colección Helmintológica del Departamento de Protozoología, Helmintología e Invertebrados Afines del Museo de Historia Natural, mientras que otros se encuentran en la Colección de Parásitos del Laboratorio de Parasitología de Fauna silvestre, Facultad de Ciencias Biológicas UNMSM.

\section{Resultados}

Del intestino de ambas tortugas también se colectaron nemátodos cuyos registros se anotarán en otro trabajo.

Como resultado del estudio se tiene que $6(75 \%)$ de $P$. expansa y $8(44,4 \%)$ de $P$. unifilis estuvieron parasitadas por individuos de una a 3 especies diferentes de Digenea.

Se identificaron las siguientes especies: Nematophila grandis (Diesing, 1839) Travassos, 1934 y Halltrema avitellina Lent \& Freitas, 1939 (Cladorchiidae Fischoeder, 1901 Schizamphistominae Looss, 1912); Podocnemitrema papillosum Alho \& Vicente, 1964 (Microscaphidiidae Looss, 1900) y Telorchis hagmanni Lent \& Freitas, 1937 (Telorchiidae Looss, 1899, Telorchiinae Looss, 1899).

La Tabla 1 resume las especies parásitas y su prevalencia según el hospedero mientras que las asociaciones de especies de digenea encontradas en cada especie de tortuga parasitada se encuentran anotadas en la Tabla 2.

\section{Nematophila grandis (Diesing, 1839) Travassos, 1934}

$$
\left(\mathrm{N}^{\circ}\right. \text { Ingreso - MUSM: 2975) }
$$

Numerosos especímenes de esta especie se obtuvieron tanto de $P$. expansa como de $P$. unifilis, todos ellos adheridos a la mucosa del estómago y en algunos casos formando túneles en la submucosa, aunque los huéspedes aparentaban condición normal. De cada animal se retiraron entre 10 y 30 individuos. Ellos miden 22,5 (22 - 24) de largo por 7,6 (7 - 8) de ancho; en algunos casos se encontraron individuos que alcanzaban los $5 \mathrm{~cm}$ de longitud. La faringe y el esófago son largos, de 5 (4,5 $-5,3)$ de longitud.

Nematophila grandis se encuentra en tortugas acuáticas de América central y del sur, y como lo han señalado Salízar y Sánchez (2004), de acuerdo a varios autores, en varias especies 
Tabla 1. Digeneos identificados en 2 especies de Podocnemis spp. procedentes de lquitos, Perú.

\begin{tabular}{cll}
\hline \multicolumn{1}{c}{ Digenea } & Hospedero & Prevalencia $\mathbf{( *}^{*}$ \\
\hline Cladorchiidae & & \\
Nematophila grandis & $\begin{array}{l}\text { P. expansa } \\
\text { P. unifilis }\end{array}$ & $2 / 8(25)$ \\
& $\begin{array}{l}\text { P. expansa } \\
\text { Halltrema avitellina }\end{array}$ & $5 / 8(62,5)$ \\
& P. unifilis & $1 / 18(5,5)$ \\
Microscaphidiidae & & \\
Podocnemitrema papillosum & P. expansa & $2 / 8(40)$ \\
& P. unifilis & $4 / 18(22,2)$ \\
Telorchiidae & & \\
Telorchis hagmanni & P. unifilis & $1 / 18(5,5)$ \\
\hline
\end{tabular}

(*) $\mathrm{N}^{\circ}$ parasitados $/ \mathrm{n}^{\circ}$ examinados $(\%)$

de tortugas de diferentes países americanos como Brasil, Ecuador, Guyana Francesa, México, Panamá y Venezuela; pero también se ha registrado en Argentina (Lunaschi \& Drago 2007). DíazUngría (1978) señala que $P$. expansa de Venezuela también es huésped de este digeneo. En el Perú, $N$. grandis ha sido previamente identificada en Podocnemis unifilis de Iquitos y de Madre de Dios (Salízar y Sánchez 2004, Sánchez et al. 2006), por lo que $P$. expansa es un nuevo huésped para el Perú.

\section{Halltrema avitellina Lent \& Freitas, 1939}

$$
\text { ( } \mathrm{N}^{\circ} \text { Ingreso - MUSM: 2976) }
$$

Esta especie mide 5,5 (5-7) de largo por 4,3 $(4-4,5)$ de ancho; los huevos operculados son grandes, de color amarillento y miden $119 \mu \mathrm{m}(115-122)$ de largo por $55 \mu \mathrm{m}(54-56,5)$ de ancho.

Jones (2005) ha considerado que tanto Cladorchis heteroxenum Cordero \& Vogelsang, 1940 como Pseudollassostoma heteroxenum (Cordero y Vogelsang, 1940) Yamaguti, 1958 son sinónimos del género Halltrema Lent \& Freitas, 1939. Esta especie se registra por primera vez para el Perú.

\section{Podocnemitrema papillosum Alho \& Vicente, 1964}

$$
\text { ( } \mathrm{N}^{\circ} \text { Ingreso - MUSM: 2977) }
$$

Mide 4,4 (4 - 5) de longitud, se caracteriza por carecer de acetábulo y tener la ventosa oral escondida por lo que la boca se comunica al exterior por medio de una excavación; de cuerpo ovalado y con papilas. Todas las demás características de nuestros especímenes coinciden con la descripción de Alho \&
Vicente (1964) y Blair (2005). Brooks (1976) ha señalado que 2 Microscaphidiidae se encuentran en Podocnemis sudamericanas, Podocnemitrema papillosum en P. expansa de Brasil y Neodeuterobaris pritchardae Brooks, 1976 en P. lewyana de Colombia; por tanto, esta es la primera vez que se registra a $P$. papillosum en $P$. expansa y $P$. unifilis en el Perú, siendo esta última especie un nuevo huésped.

\section{Telorchis hagmanni Lent \& Freitas, 1937}

$$
\text { (Col. PAS - FCB: 256) }
$$

Esta especie no es común en Podocnemis spp., siendo la prevalencia muy baja por lo que se colectaron solo 2 especímenes. Brooks (1976) encontró 1 individuo en cada una de las especies P. expansa de Brasil y P. lewyana de Colombia. Las siguientes son las principales características: cuerpo cubierto de espinas, con mayor densidad en la región anterior; miden 20 y 22 de largo por 2 y 2,3 de ancho a nivel de la parte media del cuerpo; los ciegos intestinales terminan cerca del extremo posterior del cuerpo. Las vitelógenas están formadas por folículos grandes, se localizan desde el nivel del borde posterior del ovario hasta poco antes del testículo anterior. El ovario es ovalado y los testículos dispuestos en la parte posterior del cuerpo y en tándem. Poro genital pre acetabular. Los huevos operculados tienen un reborde en la base del opérculo, in útero miden $31 \mu \mathrm{m}(29-33,5)$ de largo por $11 \mu \mathrm{m}(16-17,3)$ de ancho. Esta especie es un nuevo registro para el Perú.

Se puede advertir que las tortugas Podocnemis poseen una importante diversidad de digeneos porque en otros países sudamericanos también se han identificado varias especies además de las señaladas aquí como, por ejemplo, Braunotrema pulvinatum, Rhytidodes gelatinosus, Telorchis bifurcus, Loefgrenia loefgreni, Helicotrema spirale, Neodeuterobaris pritchardae, entre otras (Travassos et al. 1976).

\section{Literatura citada}

Alho C.J.R. \& J.J. Vicente. 1964. Podocnemitrema papillosus g. n. sp. n. e nova organização de sistemática da familia Microscaphidiidae Travassos, 1922 (Trematoda, Paramphistomoidea). Rev. Bras. Biol. 24: 17-22.

Blair D. 2005. Family Microscaphidiidae Looss, 1900. Págs. 193211. In Keys to the Trematoda. Vol. 2. D. I. Gibson, A. Jones and R. A. Bray eds. CABI Publishing, London U.K. 768 pp.

Brooks D.R. 1976. Neodeuterobaris pritchardae gen. et sp. n. (Digenea: Microscaphidiidae) in a sideneck turtle, Podocnemis lewyana Dumeril, 1852, from Colombia. J. Parasitol. 62: 426-428.

Tabla 2. Asociaciones de especies de Digeneos en 2 especies de Podocnemis spp. de Iquitos, Perú.

\begin{tabular}{lccc}
\hline Hospedero & Parasitados $\left(^{*}\right)$ & N. ${ }^{\circ}$ especies digeneos & Asociación \\
\hline P. expansa & $3 / 6(50)$ & 1 & Halltrema o Podocnemitrema \\
P. expansa & $2 / 6(33,3)$ & 2 & Nematophila + Podocnemitrema \\
P. expansa & $1 / 6(16,6)$ & 3 & Nematophila + Hallatrema + Podocnemitrema \\
P. unifilis & $5 / 8(62,5)$ & 1 & Nematophila, Podocnemitrema o Telorchis \\
P. unifilis & $2 / 8(25)$ & 2 & Nematophila + Podocnemitrema \\
P. unifilis & $1 / 8(12,5)$ & 3 & Nematophila + Halltrema + Podocnemitrema \\
\hline
\end{tabular}

(*) $\mathrm{N}^{\circ}$ parasitados según asociación $/ \mathrm{n}^{\circ}$ total parasitados $(\%)$ 
Díaz-Ungría C. 1978. Helmintos parasites de vertebrados en el estado de Zulia (Venezuela). Algunas especies nuevas para Venezuela. Veterinaria Tropical 3: 15-37.

Dyer W.G. \& J.L. Carr. 1990. Some digeneans of the neotropical turtle genus Rhinoclemmys in Mexico and South America. J. Helminthol. Soc. Wash. 57: 12-14.

Jones A. 2005. Family Cladorchiidae Fischoeder, 1901. Pags. 192-212. In Keys to the Trematoda. Vol. 2. D. I. Gibson, A. Jones and R. A. Bray eds. CABI Publishing, London U.K. 768 pp.

Lent H. \& J.F.T. Freitas. 1937. Pesquisas helminthologicas realizadas no Estado do Pará. I. Trematoda: Facioloidea. Mem. Inst. Oswaldo Cruz 32: 449-460.

Lunaschi L.I. \& F.B. Drago. 2007. Cheklist of digenean parasites of anphibians and reptiles from Argentina. Zootaxa 1476: 51-68.

Salízar P. \& L. Sánchez. 2004. Primer registro para el Perú de Nematophila grandis (Diesing, 1839) Travassos, 1934 (Trematoda, Diplodiscidae) en Podocnemis unifilis (Troschel, 1848) (Testudines, Pelomedusidae). Rev. peru. Biol. 11: $37-40$.
Salízar P. \& L. Sánchez. 2007. Nuevos registros de nematodos en dos especies de tortugas (Reptilia: Testudines) en el Perú. Neotrop. Helminthol., 1: 43-45.

Sánchez N., M. Tantaleán, D. Vela \& A. Méndez. 2006. Parásitos gastrointestinales de la taricaya, Podocnemis unifilis (Troschel, 1848) (Testudines: Podocnemididae) de Iquitos, Perú. Rev. peru. biol. 13: 119-120.

Sarmiento L., M. Tantaleán \& A. Huiza. 1999. Nemátodos parásitos del hombre y de los animales en el Perú. Rev. Peru. Parasitol., 14: 9-65.

Tantaleán M. 1998. Nuevos registros de nemátodes parásitos de animales de vida silvestre en el Perú. Rev. peru. biol. 5: 103-104.

Tantaleán M., D. Juárez \& C. Cruz. 1983. Helmintos nuevos para el Perú. Bol.Inst. Med. Trop. UNMSM., 3: 1-3.

Travassos L., J. F. T. Freitas \& A. Kohn. 1969. Trematodos do Brasil. Mem. Inst. Oswaldo Cruz, 67: 1-886. 
